\title{
Infinitesimal Idealization, Easy Road Nominalism, and Fractional Quantum Statistics
}

\author{
by Elay Shech \\ January 2018
}

Forthcoming in special issue of Synthese: Infinite Idealizations in Science

\begin{abstract}
It has been recently debated whether there exists a so-called "easy road" to nominalism. In this essay, I attempt to fill a lacuna in the debate by making a connection with the literature on infinite and infinitesimal idealization in science through an example from mathematical physics that has been largely ignored by philosophers. Specifically, by appealing to John Norton's distinction between idealization and approximation, I argue that the phenomena of fractional quantum statistics bears negatively on Mary Leng's proposed path to easy road nominalism, thereby partially defending Mark Colyvan's claim that there is no easy road to nominalism.
\end{abstract}

\section{Introduction}

The overarching goal of this essay is to draw attention to some interesting connections between the literature on infinite and infinitesimal idealizations in science and issues in philosophy of mathematics such as mathematical indispensability and explanation. ${ }^{1}$ Recently, several papers have discussed the feasibility of the so-called "easy road" to nominalism. ${ }^{2}$ The question motivating (what I will call) the easy road nominalism debate is whether or not it is possible to reject platonism about mathematical objects, while committing to some substantial form of scientific realism, but doing so without taking the "hard road" to nominalism. The hard road includes purging our best scientific theories from

\footnotetext{
${ }^{1}$ Looking ahead, I do not intend to overstate my case. Some connection have already been made, e.g., Maddy (1997), Leng (2010), Baron (2016), De Bianchi (2016). Nevertheless, the case study that I consider, the relevance of Norton's distinction between approximation and idealization, and the particular problems posed by infinite/infinitesimal idealizations for issues in philosophy of mathematics have been largely ignored to my best knowledge.

2 E.g., Colyvan (2010, 2012), Azzouni (2012), Bueno (2012), Leng (2012), Liggins (2012), and Yablo (2012). Also see even more recent essays in the special issue Molinini et al. (2016).
} 
quantifying over abstract mathematical objects. ${ }^{3}$ Said differently, advocates of the hard road to nominalism claim that mathematical objects are not indispensable to our best scientific theories, and then continue to dispense with such objects. They then are not vulnerable to the Quine-Putnam indispensability argument, a main tenet of which is the Quinean ontic thesis that we are committed to the existence of all the entities we indispensably quantify over in our best scientific theories. ${ }^{4}$ The easy road nominalists, on the other hand, accept the thesis that mathematical entities are indispensable to our best scientific theories. Nevertheless, it is claimed that indispensability does not warrant reification (at least in the case of mathematical entities).

Various strategies have been explicated on behalf of the easy road, ${ }^{5}$ but Colyvan (2010) argues that such approaches can only succeed if one rejects the idea there are genuine mathematical explanations of empirical phenomena in science. However, many proponents of the debate agree that this seems unlikely. ${ }^{6}$ What I wish to do here is concentrate on easy road nominalism that embraces scientific realism, accepts that there are mathematical explanations in science, and is thus moved by what Alan Baker $(2009,6130)$ has called the Enhanced Indispensability Argument (EIA):

(1) We ought rationally to believe in the existence of any entity that plays an indispensable explanatory role in our best scientific theories.

(2) Mathematical objects play an indispensable explanatory role in science.

(3) Hence, we ought rationally to believe in the existence of mathematical objects. ${ }^{7}$

\footnotetext{
${ }^{3}$ See Field (1980) for such an attempt.

${ }^{4}$ See Putnam (1971) and Quine (1981), and see Colyvan (2001) for an in-depth study of the indispensability argument and a defense.

${ }^{5}$ E.g., Azzouni (2004),Balaguer (1998), Melia (2000, 2002), and Yablo (1998, 2002).

${ }^{6}$ Mainly because of numerous examples that do seem to provide genuine mathematical explanation, e.g., Baker $(2005,2009,2012)$, Colyvan $(2001,2007,2010)$. Moreover, the example discussed in this paper purports to be such a genuine mathematical explanation.

${ }^{7}$ Many are unconvinced by the EIA and I would like to say a bit about why some may feel the argument is compelling. Science is in the business of giving scientific explanations, but it is hard to see how something can be explanatory but not exist. For example, on a causal-mechanistic account of explanation (e.g., Salmon 1984), it would seem absurd if one were told that a particular gene mutation explains some disease but that the same gene doesn't exist. Similarly, on a covering law account of explanation (Hempel and Oppenheim 1948) it seems unreasonable to explain the motion of planets given Newton's universal law of gravitation but then claim that there is no such thing as the universal law of gravitation. In the same manner, Platonists like Colyvan (2001, 2007, 2010) and Baker $(2005,2009,2012)$ think that there is something seriously wrong with denying the existence of, say, prime numbers, but then arguing that the primeness of thirteen and seventeen (along with a number of evolutionary hypothesis) explains why the North American periodical magicicada cicadas only appear every thirteen or seventeen years. Of course, there are others who think that fiction can be explanatory, e.g. Bokulich (2008). In any case, in the context of this paper I will assume that the EIA is convincing enough to take seriously and ask whether Leng's $(2005,2010,2012)$ approach to evade the arguments works.
} 
Such an approach is put forth by Mary Leng $(2005,2010,2012)$. In this paper, I will argue that a particular example from mathematical physics, so-called "fractional quantum statistics" manifested by "anyons" in "fractional quantum Hall effect" systems, bears negatively on Leng's proposal for easy road nominalism, and in doing so I partially defend Mark Colyvan's (2010, 2012a, 2012b) claim that there is no easy road to nominalism. ${ }^{8}$ What is interesting about the anyon case study is that it appeals to a kind of infinitesimal idealization-a two-dimensional model of quantum mechanics-that in turn seems to play an essential role in the scientific account of anyons. This is similar to other infinite idealizations, such as thermodynamic-type limits for phase transitions and spontaneous symmetry breaking (Batterman 2002, 2005; Ruetsche 2011; Shech 2013), and infinitesimal idealizations, such as in the context of the quantum-classical limit (Batterman 2002, Bokulich 2008), which purportedly play an indispensible explanatory role. ${ }^{9}$ Thus, in fleshing out the consequences of fractional quantum statistics for Leng's approach to easy road nominalism, I am attempting to fill a lacuna in the easy road nominalism debate. ${ }^{10}$ My suggestion then is that further insight into the plausibility of easy road nominalism may be gained by studying closely examples from science wherein ostensibly essential, infinite/infinitesimal idealizations arise.

In what follows, I first introduce Leng's $(2005,2010,2012)$ approach to easy road nominalism in Section 2 and show how it depends on the idea that a physical system can "approximately instantiate" mathematical structure where a mathematical explanation is at hand. Since Leng $(2005,2010,2012)$ does not does explicate what is meant by "approximate instantiation," I critically consider a possible way of expounding on her account via John Norton's (2012) well-received distinction between approximations and idealizations in Section 3: physical structure can approximately instantiate

\footnotetext{
${ }^{8}$ In hope to thwart an objection early, note that I'm only partially defending Colyvan (2010) since there are other approaches to easy road nominalism, e.g., Azzouni (2012), Bueno (2012), Liggins (2012), and Yablo (2012), which purport to make sense of mathematical explanation. Also recall that the overarching goal of this paper is to make interesting connections between infinite and infinitesimal idealization in science and the easy road nominalism debate-it is not to mount a comprehensive defense of Colyvan's (2010) thesis.

${ }^{9}$ There may be an interesting difference between infinite and infinitesimal idealizations but for the purpose of this paper I'm assuming that both idealizations are on equal footing, so to speak. Note that, insofar as an idealization enters physical theory via a limiting procedure, an infinite idealization is just the inverse of an infinitesimal idealization and vice versa.

${ }^{10}$ Very recently, the role of idealizations in the debate have been considered by Baron (2016) and De Bianchi (2016), which I discuss briefly in Section 5, but the emphasize in not placed on the distinctly indispensable role of infinite/infinitesimal idealization in science. Similarly, discussion of idealization in the philosophy of mathematics, e.g., Maddy (1997), Leng (2010; 116-122, 133-137), either does not explicitly discuss the type of essential, infinite/infinitesimal idealizations that I'm concerned with; or else it is assumed or concluded that such issues do not introduce substantive novel problems for the nominalist. I will argue otherwise.
} 
mathematical structure if the latter can be characterized as an approximation (in Norton's sense) of the former. Next, I present the case study of the anyons and fractional statistics along with the standard explanatory story found in the physics literature in Section 4 as an example of an indispensable mathematical explanation wherein there is no sense in which the mathematical structure appealed to in order to explain fractional statistics is "approximately instantiated" in a physical system. Section 5 explains that cases such as fractional statistics are a common feature of our best physical theories and entertains three nominalist objections. My hope is that it will be clear from my discussion of fractional statistics and essential, infinite/infinitesimal idealizations that the study of such examples may greatly inform the debate on easy road nominalism and, more generally, the cogency of mathematical explanations. I conclude the paper with a short summary in Section 6 (and have added an appendix at the end of the paper with technical details).

\section{Leng's Easy Road Nominalism}

Mary Leng's $(2005,2010,2012)$ approach to easy road nominalism takes the form of a defense of nominalistic scientific realism (NSR), which may be characterized as follows:

The view that the nominalistic content of empirical science-that is, what empirical science entails about the physical world-is true (or mostly true...), while its platonistic content-that is, what it entails "about" an abstract mathematical realm-is fictional. (Balagaur 1998, 131)

There is a rough consensus that the plausibility of NSR depends on either excising mathematical explanations from science, or else illustrating "how mathematics can be expected to function successfully in explanatory contexts even if mathematical objects are taken to be mere fictions" (Leng $2012,986)$. The later route, taken by Leng $(2005,2010,2012)$, involves showing that mathematical structure plays a representational role (in the mathematical explanation of a physical phenomenon) such that "...our explanations work by displaying the phenomenon as being a consequence of the (approximate) instantiation of that structure in the empirical situation at hand" (Leng 2012, 990-991).

In other words, we can avoid platonism but remain scientific realists (of the NSR bent) by showing how an explanation of physical phenomenon is given in virtue of the fact that the phenomenon follows from a mathematical structure that is approximately instantiated in a physical 
system. These types of explanations, called structural explanations by Leng, are fundamentally mathematical explanations. ${ }^{11}$ However, the fact that the physical system approximately instantiates the mathematical structure allows us to gain a genuine mathematical explanation without reification.

A structural explanation will explain a phenomenon by showing (a) that the phenomenon occurs in a physical system instantiating a general mathematical structure, and (b) the existence of that phenomenon is a consequence of the structure characterizing axioms once suitably interpreted. (Leng 2012, 989)

As an example, Leng $(2012,989-990)$ considers the explanation of why it is impossible to construct a square with the same area of a circle (with only a compass and ruler). The reason is that $\pi$ (and thus $\sqrt{\pi}$ ) is a transcendental number. ${ }^{12}$ The explanation consists of two parts. First, (a) physical space approximately instantiates the structure of Euclidean space (and distance between points in Euclidean space approximately instantiates the real numbers structure). Second (b), once we define notions like rule/compass constructions and constructible points, we can show that it follows from the axioms of Euclidean geometry that we cannot construct transcendental (real) numbers.

Thus, the ultimate applicability of this explanation comes, not from the existence as abstract of any of the mathematical objects involved, but rather, from the interpretation of the geometrical axioms as (approximately) true about the physical space of paper and pencil drawings... [T]he explanation is explanatory because it shows an empirical phenomenon (our inability to find certain ruler/compass constructions) to result from the general structural features of the situation. It enables us to see the relation between the impossibility of circle squaring and other impossible constructions, and it enables us to see the phenomenon as resulting from a necessity not just from bad luck or poor construction. (Leng 2012, 990 )

\footnotetext{
${ }^{11}$ In calling such explanations structural explanation Leng is following some previous authors, e.g., Railton (1980), Hughes (1989), Bokulich (2008). For instance, according to Bokulich $(2008,149)$ :
}

[A] structural explanation is one in which the explanandum is explained by showing how the (typically mathematical) structure of the theory itself limits what sorts of objects, properties, states, or behaviors are admissible within the framework of that theory, and then showing that the explanandum is in fact a consequence of that structure.

${ }^{12} \mathrm{~A}$ transcendental number is a number that is not a root of a (non-trivial) polynomial with rational coefficients (where a rational number is any number that can be expressed as a fraction of two integers). 
Leng's account, although intuitive and attractive, suffers from the weakness that the notion of "approximate instantiation" is vague and not well defined. Accordingly, in the following section I will attempt to remedy the situation by appealing to John Norton's (2012) well-received distinction between "approximation" and "idealization."13

\section{Approximation, Idealization, and Approximate Instantiation}

John Norton $(2012,209)$ characterizes the difference between "approximation" and "idealization" as follows: ${ }^{14}$

An approximation is an inexact [representation] of a target system. [...]

An idealization is a real or fictitious system, distinct from the target system, some of whose properties provide inexact [representations] of some aspects of the target system.

More specifically, we can think of approximations and idealizations entering physical theory through limiting procedures in which we consider sequences of systems (or models). The general scheme that is implemented is as follows (212):

System ${ }_{1}$, System $_{2}$, System $_{3}, \ldots \quad \rightarrow$ Limit System

And the corresponding sequence of properties is:

Property $_{1}$, Property $_{2}$, Property $_{3}, \ldots \rightarrow$ Limit Property

A limit property is an approximation (of, say, System ${ }_{1}$ 's property Property ${ }_{1}$ ) in Norton's sense and it has to do with the property of a system and its limit, i.e., a limit of the properties of a sequence of systems. No novel reference is made to a real or fictitious system. Rather, the target system is referred to directly and represented inexactly. On the other hand, a limit system is an idealization in Norton's

\footnotetext{
${ }^{13}$ For the purposes of this paper I set aside the objection that there is no such thing as "physical structure" (and thus no such thing as a physical system instantiating any kind of mathematical structure) since "structure" is, strictly speaking, a set-theoretic notion (Frigg 2006).

${ }^{14}$ For well-received characterizations and taxonomies of idealizations see McMullin (1985) and Weisberg (2013).
} 
sense, and it concerns a system and its limit, i.e., a limit of a sequence of systems. ${ }^{15}$ Sometimes the limit property (i.e., a Nortonian approximation) is equal to the corresponding property of the limit system (i.e., the property of the Nortonian idealization). If, in turn, said limit system is used to represent some physical target system, then it makes sense to say that the property of the limit system is approximately instantiated in the target system. However, sometimes a limit property is not equal to the property of the limit system and in such context it is not the case that the property of the limit system is approximately instantiated in the target system. Thus, in brief, we can say that a physical structure approximately instantiates mathematical structure when the mathematical structure is an approximation (in Norton's sense) of the physical structure.

In a bit more detail, but still schematically, my proposal to expound on Leng's account using Norton's distinctions is as follows. Let some real physical system be faithfully and accurately represented by (some abstract-mathematical system) $S_{i}$, where $S_{i}$ has a structure characterized by a set of properties $\left\{P_{i}^{R}\right\}$. ${ }^{16}$ Let some abstract and idealized limit system $S_{\infty}$, with a mathematical structure characterized by a set of properties $\left\{P_{i}^{\infty}\right\}$, represent or model $S_{i}$ in some explanatory context. The notion of approximate instantiation can be fleshed out in the following manner: A physical system $S_{i}$ approximately instantiates a general mathematical structure $\left\{P_{i}^{\infty}\right\}$ just in case the limit property, symbolized by $\lim _{i \rightarrow \infty} P_{i}^{R}$, equals the corresponding property of the idealized limit system $S_{\infty}$, viz., $P_{i}^{\infty}=\lim _{i \rightarrow \infty} P_{i}^{R}$, for every property $i$ in the set characterizing the structure $\left\{P_{i}^{\infty}\right\}$ to be instantiated. Alternatively, we could concentrate specifically on properties and say that a physical system $S_{i}$ instantiates a mathematical property $P_{i}^{\infty}$ when $P_{i}^{\infty}=\lim _{i \rightarrow \infty} P_{i}^{R}$ (i.e., when the property $P_{i}^{\infty}$ of the limit system $S_{\infty}$ matches the corresponding limit property $P_{i}^{\infty}$ ). I am thus suggesting a kind of condition of

\footnotetext{
${ }^{15}$ Butterfield (2011, Section 3) discusses similar distinctions. In particular, he makes a distinction between a system $\sigma(N)$ that depends on some parameter $N$ (let $\{\sigma(N)\}$ denote a sequence of such systems), a quantity defined on the system $f(\sigma(N))$ (let $\{f(\sigma(N))\}$ denote a sequence of quantities on successive systems), and a (real number) value $v(f(\sigma(N))$ ) of quantities on successive systems (where a sequence of states on $\sigma(N)$ is implicitly understood; let $\{v(f(\sigma(N)))\}$ denote a sequence of values on successive systems). A limit system $\sigma(\infty)$ arises when $\lim _{N \rightarrow \infty}\{\sigma(N)\}$ is well-defined-otherwise there is no limit system. A property of a limit system refers to the value $v(f(\sigma(\infty))$ of the (natural) limit quantity $f(\sigma(\infty))$ (in the natural limit state) on $\sigma(\infty)$. A limit property $v(f(\sigma(N))$ is a limit of a sequence of values of quantities on successive systems (or, values on the systems on the way to the limit) and is well-defined when $\lim _{N \rightarrow \infty}\{v(f(\sigma(N)))\}$ exists. The question that I am discussing is whether a property of a limit system equals the system's limit property since this is what it precisely means for a property to be approximately instantiated. Specifically, the question asks whether $v\left(f(\sigma(\infty))=\lim _{N \rightarrow \infty}\{v(f(\sigma(N)))\}\right.$ (assuming both are well-defined).

${ }^{16}$ Strictly speaking, a structure $S=\langle U, O, R$,$\rangle is a composite entity consisting of a non empty set U$ of individuals called the domain of the structure $S$, an indexed set $O$ of operations of $U$, and a non-empty indexed set $R$ of relation on $U$, where we can think of properties as one-placed relations. For my purposes discussing structure as a set of properties will do. In principle, one can extend my claims to the more formal characterization of structure.
} 
adequacy on any Leng-type NSR account of a phenomenon appearing in some physical target system, which appeals to the notion of "approximate instantiation:"

Condition of Adequacy: Approximate instantiation can adequately justify an NRS account of a phenomenon appearing in a physical target system just in case there is a match between the relevant properties of the limit system (using to represent/model the target system) and the corresponding limit properties. ${ }^{17}$

The problem for Leng's proposed easy road nominalism arises when we have a mathematical explanation appealing to a structure that cannot be approximately instantiated in the physical situation at hand so that the above stated condition of adequacy is not satisfied, i.e., there is a mismatch between some (used for explanatory purposes) property of a limit system and its corresponding limit property. In the following section I will present an example that I submit is a genuine mathematical explanation, but where there is no precise sense in which the mathematical structure is approximately instantiated in the physical world. In the rest of this section I will attempt to further elucidate said claims with a toyexample. Readers familiar with Norton's (2012) distinction (or Butterfield's (2011) analogous distinction) are encouraged to skip ahead to the next section.

Envisage some physical system with physical structure faithfully represented by a threedimensional cube with sides of length $L$ and with some impenetrable point-like object in its interior, known as a "hole" (see Fig. 1). We'll be interested in two properties concerning this system. First, whether or not loops inside the cubical space can be continuously deformed into a point. If this is possible we'll say that (the topology of) the space is simply connected and that it has a trivial fundamental group (see Fig. 2). If not, we'll say the space is non-simply connected (or multiply connected) and that it has a non-trivial fundamental group (see Fig. 3). ${ }^{18}$

\footnotetext{
${ }^{17}$ One may worry that the characterization for "approximate instantiation" suggested here seems to privilege "continuous" properties over "discrete" properties. I do not think that this is the case. We can reasonably talk about a discrete series converging to a number, e.g., $1, \frac{1}{2}, \frac{1}{4}, \frac{1}{8}, \ldots \rightarrow 0$, or even a sequence of non-continuous functions converging to a function, e.g., take $f(x)=0$ if $x \neq 0$ and $f(x)=\frac{1}{n}$ if $x=0$. Foreshadowing what is to come, the problem with topological properties like "connectedness" is that, first, there is no convergence and, second, notions like convergences, limits, continuity, etc., all presuppose topological properties.

${ }^{18}$ These are all rough and intuitive characterizations of the notions of connectedness, homotopy, etc. They will do for my purposes, and I will only introduce further details when necessary. For precise characterization see standard textbooks on algebraic topology, e.g. Hatcher (2002).
} 
Second, we'll want to ascertain what is the (three-dimensional notion of a) volume $V$ of the cubical system. Clearly, our cubical system has a volume equal to $L^{3}$ and (as can be seen in the Fig. 4) its space is simply connected (i.e., it has a trivial fundamental group) since all loops-even those that lie in the plane with the impenetrable hole-can be continuously deformed and shrunk to a point.

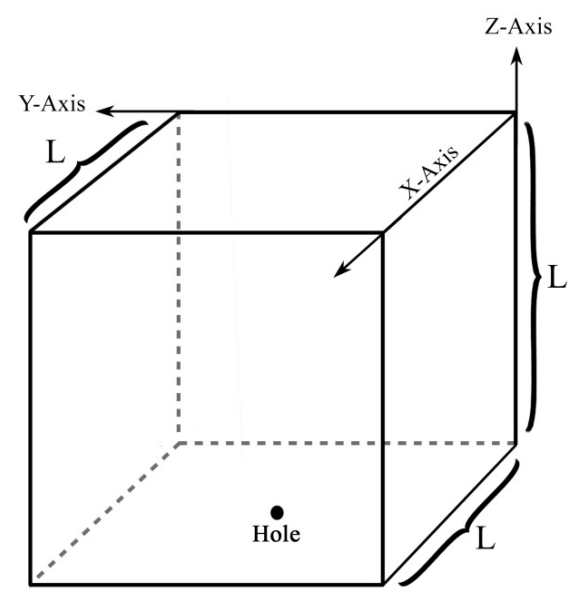

Fig. 1 3D cubical space with a hole (an impenetrable obstruction).

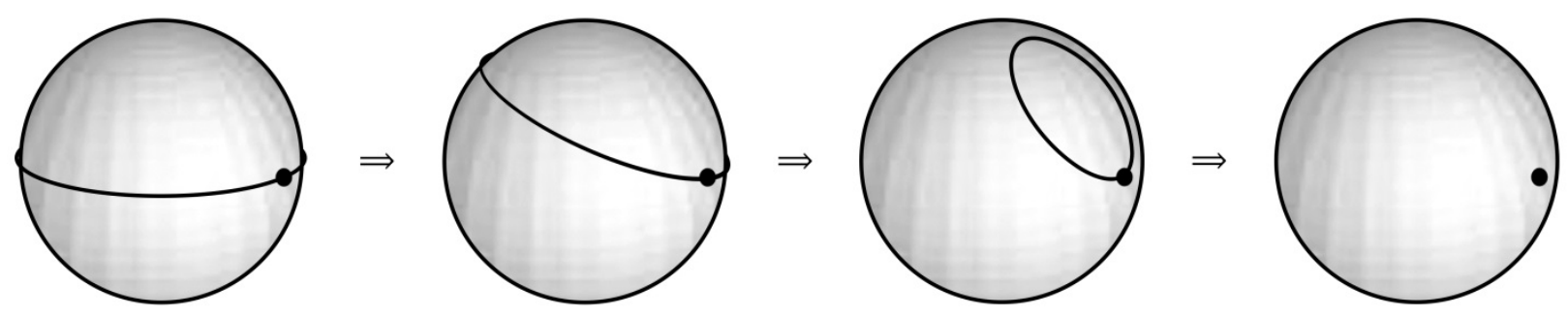

Fig. 2 The surface of a sphere as a simply connected space with a trivial fundamental group.

Next, consider a sequence of such systems in which the height of the cuboids, denoted by $l$, shrinks (see Fig. 5). That is to say, we'll consider the sequence in the limit in which $l \rightarrow 0$ (i.e., an infinitesimal idealization-approximation). So long as we are on the way to the limit system, each system in our sequence will have volume equal to $L^{2} l$ and trivial fundamental group. However, at the limit our limit system will be a two-dimensional square with a zero volume and non-trivial fundamental group (see Fig. 6). That is to say, loops surrounding the hole cannot be continuously deformed to a point without being obstructed by the impenetrable hole (see Fig. 7). 

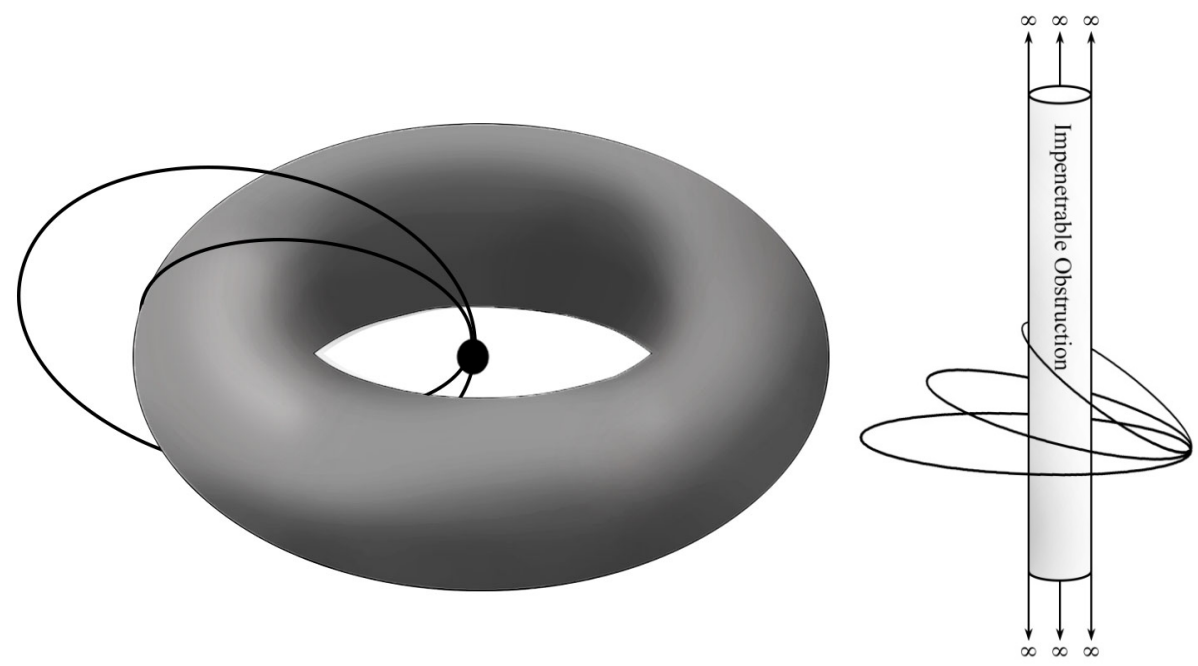

Fig. 3 Non-simply connected space with a non-trivial fundamental group in which some loops cannot be continuously shrunk to a point in the context of an impenetrable torus (left) and an impenetrable infinite cylinder (right).
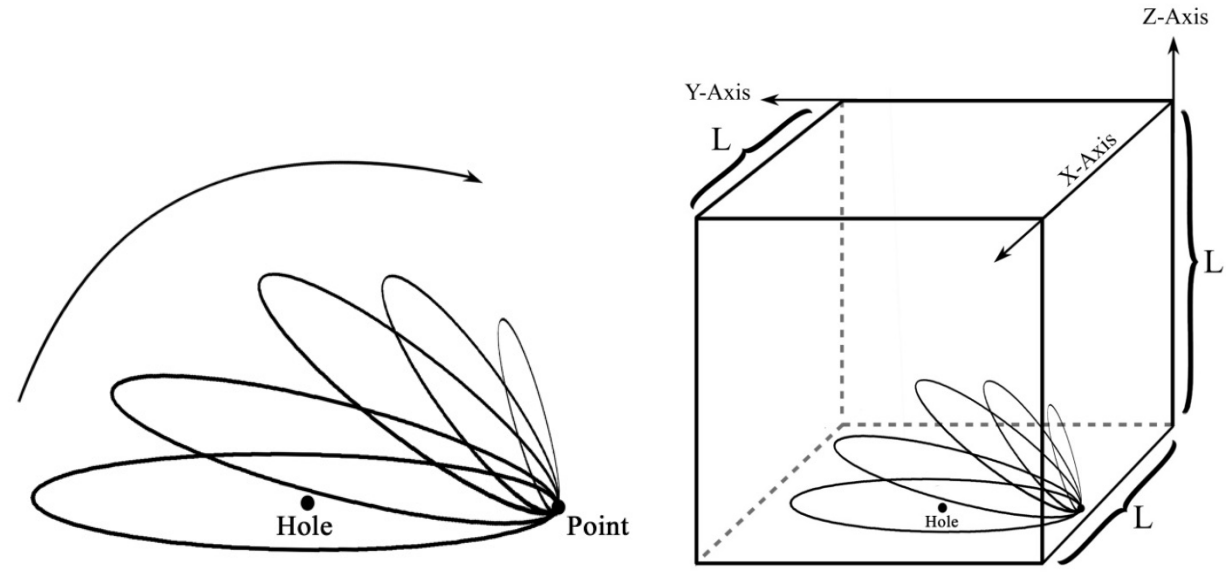

Fig. 4 (Left) A simply connected space with a trivial fundamental group in which loops can be continuously deformed to a point. (Right) A cubical space that is simply connected and has a volume of $V=L^{3}$. 


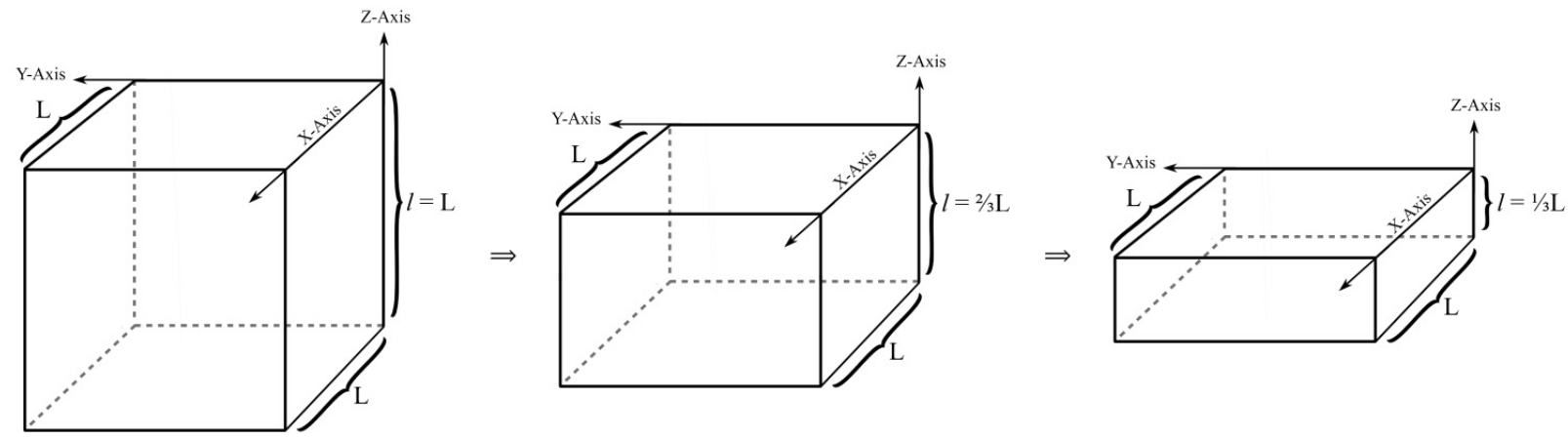

Fig. 5 A sequence of systems of cuboids as the height shrinks.

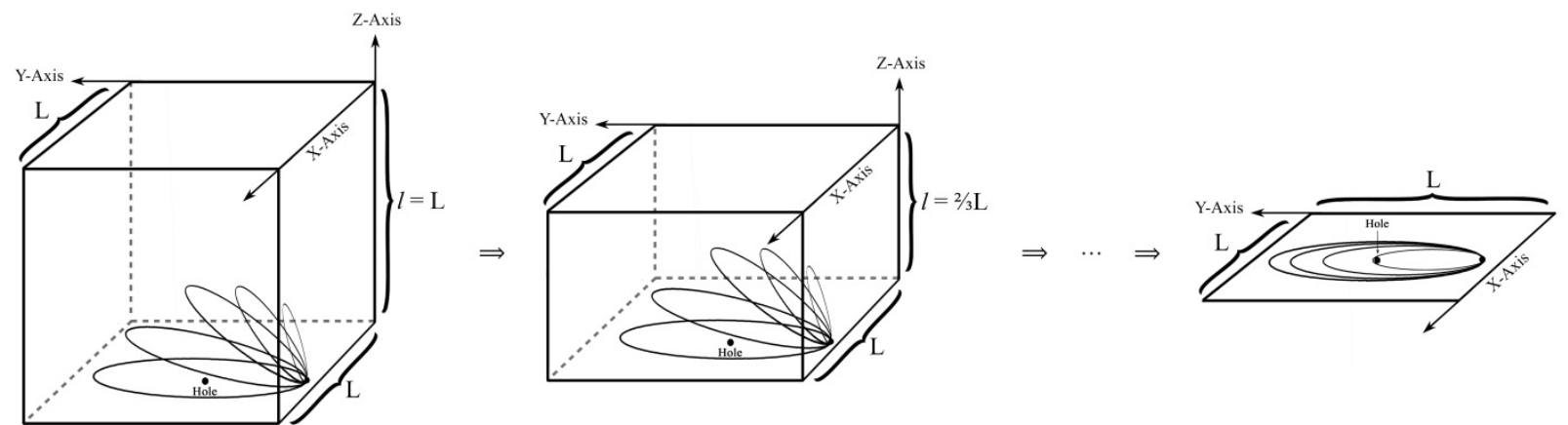

Fig. 6 On the way to the limit, each system in the sequence of cuboids is simply connected with trivial fundamental group, and has a non-zero volume. At the limit, the system is multiply connected with a non-trivial fundamental group, and with a zero volume.

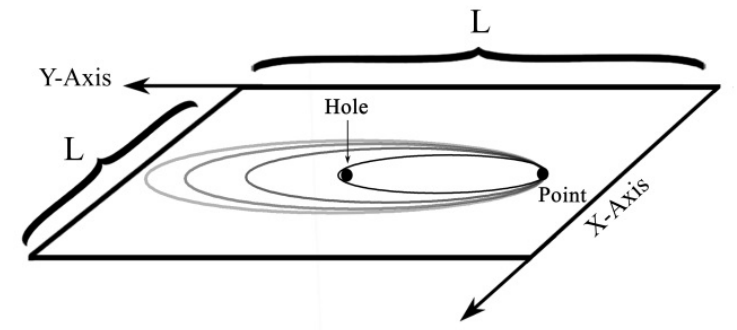

Fig. 7 At the limit, the system is a two-dimensional square and so the space is multiply connected with a non-trivial fundamental group because loops surrounding the hole cannot be shrunk to a point. 
Concentrating on the volume of our system we see that the limit property $V=L^{2} l$ corresponds to the property of the limit system $V=0$ when we take the limit. Said differently, if we 'de-idealized' away by a small epsilon $\varepsilon$ amount from the limit system so that the height $l=\varepsilon$, then the volume will be $V=L^{2} \varepsilon$ and this is approximately equal to zero. ${ }^{19}$ Accordingly, we can say that a real physical system approximately instantiates the structure of our idealized limit system with respect to the property of volume since there exist a correspondence between the limit property (the approximation) and the property of the limit system (the idealization)-our condition of adequacy is satisfied. In other words, if the height of our cube is very small, its volume will be approximately zero and Leng's $(2010,2012)$ talk of "approximately instantiated" structure makes sense.

In stark contrast, when we focus upon the topology of the space we notice that the condition of adequacy fails: there is a lack of correspondence between the limit property, i.e., a trivial fundamental group and a simply connected space, and the property of the limit system, i.e., a non-trivial fundamental group and a non-simply connected space. We cannot say that our cubical space is "approximately" nonsimply connected on the way to the limit system, or that it has an "approximate" fundamental group. Instead, the space will be simply connected, and the fundamental group trivial, full stop. It is only at the limit that the property of non-simply connectedness and non-trivial fundamental group arises. Connectedness is a binary topological property. Either the space is connected or it is not; there is no intermediate. Hence, it is not the case that any realistic physical system with some non-zero height $l$ approximately instantiates the structure of our idealized limit system with respect to the property of topological connectedness since there does not exist a correspondence between the limit property (the approximation) and the property of the limit system (the idealization).

\section{Easy Road Nominalism Meets Anyons}

The purpose of this section is to present a case study from mathematical physics which is a concrete instance of a mathematical explanation-in fact, a structural explanation à la Leng $(2012,989)$ - of empirical phenomenon that bears negatively on Leng's proposed pathway to easy road nominalism. I will first introduce the standard explanatory story found in the physics literature of the emergence of anyons and their quantum statistics in Section 4.1, and then discuss the associated philosophical issues vis-à-vis mathematical explanation and indispensability in Section 4.2. Looking ahead, the explanandum

\footnotetext{
${ }^{19}$ What do we mean by "approximately" here? We mean that we can have the volume $V=L^{2} \varepsilon$ be as small as we want by choosing an appropriate epsilon so that we can come as close to zero volume as we may want.
} 
in question will be the phenomena of fractional quantum statistics manifested by quasi-particles, known as anyons, in systems such as ones manifesting the fractional quantum Hall effect (FQHE). The explanans will include an appeal to abstract mathematical structure, viz., the Braid group as the fundamental group of the wavefunction configuration space, which cannot be nominalized away through the notion of approximate instantiation. The reason being that there will be a mismatch between the relevant limit property and the corresponding property of the limit system so that our condition of adequacy will fail.

\subsection{The Configuration Space Approach to Anyons and Fractional Quantum Statistics}

Consider a collection of non-interacting, identical particles in thermal equilibrium. What are the possible ways that such a collection may occupy a set of available discrete energy states? Roughly, quantum and statistical mechanics tell us that there are two such ways, and that the expected number of particles in some specific energy state will depend of the type of particle at hand. Bosons manifest a behavior consistent with Bose-Einstein statistics, while fermions distribute themselves according to Fermi-Dirac statistics. This division into particle types, along with corresponding statistics may be captured by what has become known as the symmetrization/anti-symmetrization postulate: "The states of a system containing $N$ identical particles are necessarily either all symmetrical or all antisymmetrical with respect to permutation of $N$ particles" (Messiah (1962), p. 595). ${ }^{20}$ That is to say, if a collection of $N$ identical particles is represented by the quantum state $\Psi_{(1,2, \ldots, N)}$ and the same collection with, say, particles 1 and 2 permuted is represented by $\Psi_{(2,1, \ldots, N)}$, then the symmetrization/anti-symmetrization postulate tells us that state must be related in the following manner:

$$
\Psi_{(2,1, \ldots, N)}=e^{i \theta} \Psi_{(1,2, \ldots, N)}
$$

where the exchange phase $\theta$ can take on a value of $\theta=0$ for a system of bosons with a corresponding phase factor $e^{i \theta}=+1$ and a symmetric quantum state, or it can take a value $\theta=\pi$ for a system of fermions with a corresponding phase factor of $e^{i \theta}=-1$ and an antisymmetric quantum state.

There are two fundamental frameworks for understanding permutation invariance in quantum mechanics, which ground the symmetrization/anti-symmetrization postulate and its consequences, namely, that there are two basic types of particles and quantum statistics. Following Landsman (2016)

\footnotetext{
${ }^{20}$ See Earman (2010) for a discussion.
} 
we will call the first, due to Messiah and Greenberg (1964), the operator framework, and the second, due to, among others, Laidlaw and DeWitt (1971), Leinaas and Myrheim (1977), the configuration space framework. Landsman (2016) has argued that, in dimensions greater than two, both frameworks are equivalent and give equivalent verdicts regarding possible particle types and statistics. However, it turns out that in two dimensions the configuration space framework allows the exchange phase to take on any value. This permits the framework to represent bosons and fermions, as well as other particles known as "anyons," which are said to exhibit "fractional quantum statistics" or "intermediate quantum statistics." $^{21}$

Recall, the manner by which a collection of identical particles occupies energy states will depend on the kind of quantum statistics that such a collection manifests, which in turn depends on the type of particle considered. Particle type is decided by how such a collection behaves under permutation, and such behavior is captured by the value of the exchange phase $\theta$ and the corresponding phase factor $e^{i \theta}$. In short, on the configuration space framework, two central theorems (see Appendix) dictate that the phase factor $e^{i \theta}$ is equivalent to the one-dimensional unitary representation $\gamma$ of the fundamental group $\pi_{1}$ of the configuration space $Q$ of the collection of identical particles, symbolized by $\gamma=e^{i \theta} \cdot{ }^{22}$ It has been shown by Artin (1947), Fadell and Neuwirth (1962), and Fox and Neuwirth (1962) that the fundamental group for the two-dimensional $(d=2)$ and three-dimensional $(d=3)$ cases are given by:

$$
\begin{aligned}
& \pi_{1}(Q)=B_{N} \text { for } d=2 \\
& \pi_{1}(Q)=S_{N} \text { for } d=3
\end{aligned}
$$

where $S_{N}$ is the permutation group and $B_{N}$ is the Braid group. In other words, in three dimensions the fundamental group of the configuration space is the (finite and discrete) permutation group $S_{N}$ which admits of the known one-dimensional unitary representation: $\gamma= \pm 1$ ( +1 for bosons and -1 for fermions). In two-dimensions, on the other hand, the fundamental group is the (infinite and discrete) braid group $B_{N}$ with one-dimensional unitary representations giving rise to phase factors of the form:

\footnotetext{
${ }^{21}$ The name is due to Noble laureate Frank Wilczek (1982). Note that anyons and fractional statistics have nothing to do with so-called paraparticles and parastatistics (which arise from higher dimensional representations of the permutation group). For more on anyons see Wilczek (1990), Khare (2005), and references therein.

${ }^{22}$ See Hatcher (2002) for relevant background in algebraic topology. Roughly, the "one-dimensional unitary representation" will allow us to represent groups with numbers. The "fundamental group," also known as the first homotopy group, is a topological invariant that allows one to classify topological spaces according to whether paths or loops in the space can be continuously deformed into each other.
} 
$\gamma_{(\theta)}=e^{i \theta}$ where $0 \leq \theta \leq 2 \pi$ so that the exchange phase can take on a continuous range of factors allowing for bosons, fermions, and anyons.

In the following sub-section I will explain how the braid group, although an essential mathematical element in the explanation of fractional statistics, cannot be approximately instantiated in a physical system. In the rest of this subsection I will discuss the empirical phenomenon that calls for the emergence of anyons and fractional statistics.

When a thin current carrying conductor is subjected to a perpendicular magnetic field $\boldsymbol{B}=$ $(0,0, B)$, there exists a Lorentz force due to the magnetic field acting on the current, which induces an electric field and gives rise to a novel voltage difference, dubbed the "Hall voltage" $V_{H}$. This is known as the Hall effect, discovered by Edwin Hall in 1879 (see Fig. 8). Classically, and roughly, through Ohm's law $R=V / I$ (where $R$ is the resistance, $V$ voltage difference and $I$ the electric current), we expect the traverse resistance $R_{x y}$, also known as the Hall resistance $R_{H}=V_{H} / I$, to vary linearly with the applied magnetic field according to $R_{H}=B / e N$ (where $e$ the electron charge and $N$ is the number of electrons per unit area) and for there to be some non-vanishing longitudinal resistance $R_{x x}$. Contrary to classical expectation, experiments due to von Klitzing et al. (1980) and Tsui et al. (1982) have shown that (i) the Hall resistance is quantized and exhibits plateaus at, and that (ii) the longitudinal resistance vanishes for, values of $R_{H}$ given by $R_{H}=h / e^{2} v$ where $h$ is Planck's constant (see Fig. 9). The dimensionless number $v$-the so-called "filling factor," which describes a ratio of filled to vacant electron states-has either integer or fractional values. The former case is known as the integral quantum Hall effect (IQHE) and the latter case is known as the FQHE. ${ }^{23}$ What matters most for our purposes is that the mechanism of localization, in the form of quasiparticles that interacting with impurities, is appealed to in order to explain (i) in the FQHE. Importantly, it has been shown by Avoras et al. (1984) that such quasi-particles have fractional quantum statistics and thus are anyons. Recent experimental observations include Camino et al. (2005).

\subsection{Explaining Fractional Quantum Statistics}

What explains the emergences of anyons, i.e., particles obeying fractional quantum statistics, and is this an example of a mathematical explanation? The standard story found in the physics literature

\footnotetext{
${ }^{23}$ For more on the quantum Hall effect see Chakraborty and Pietilinen (1995), Fradkin (2013, Ch. 13), Ezawa (2013) and Stern (2008), and for recent philosophical assessments see Bain $(2013,2016)$, Guay and Sartenaer (2016a, 2016b), Lancaster and Pexton (2015), Lederer (2015), and Shech (2015).
} 
is that it is in virtue of the fact that the fundamental group of the configuration space of identical particles in two dimensions is the braid group $B_{N}$, and not the permutation group $S_{N}$, that we can have anyons and fractional statistics. ${ }^{24}$

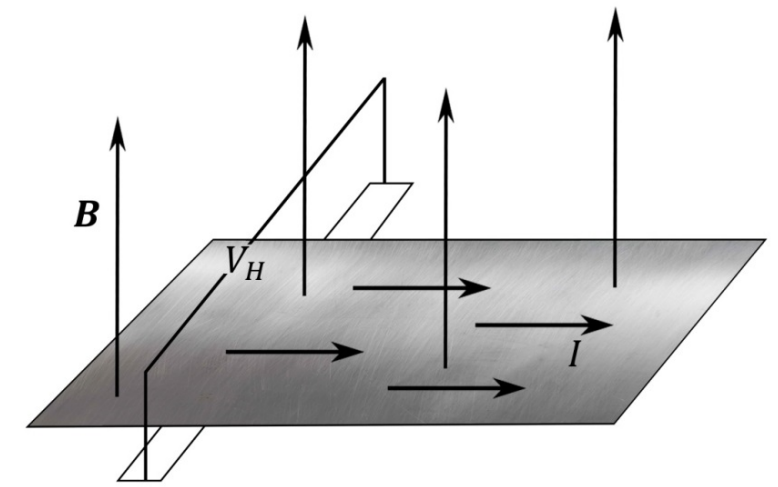

Fig. 8 The Hall effect. A current $I$ runs through a thin metal conductor in the presence of a uniform magnetic field $\boldsymbol{B}$. There exist a novel voltage drop $V_{H}$ that can be experimentally observed and varies linearly with the magnetic field.

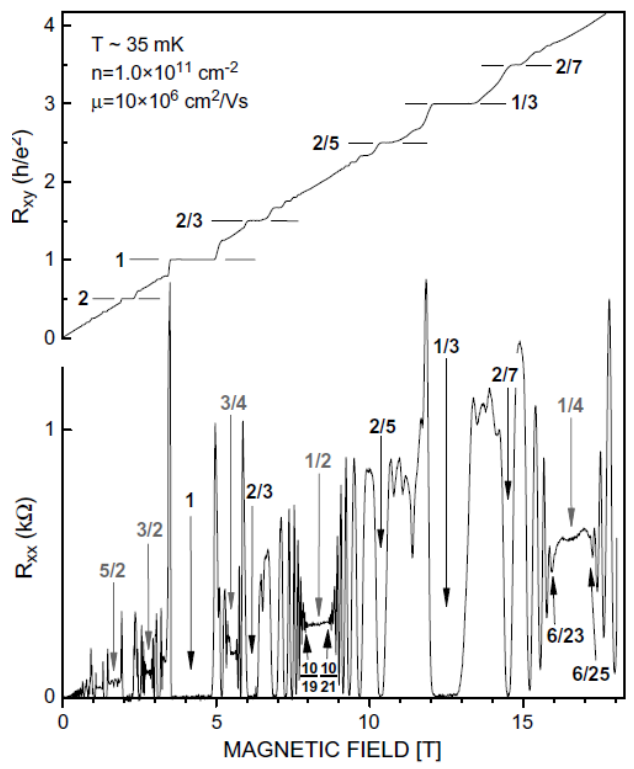

Fig. 9 Hall resistance $R_{H} \equiv R_{x y}$ and longitudinal resistance $R_{x x}$ as a function of applied magnetic field. From Stern $(2008,207)$.

\footnotetext{
${ }^{24}$ For example, “...in two dimensions, the space is multiply connected which results in the possibility of ... intermediate statistics" (Khare 2004, 5; original emphasis). By "intermediate statistics" the author is referring to "fractional statistics," and by "multiply connectedness" of space is meant "non-simply connected" space. Details are provided in the appendix, but a mere glance at the literature (e.g., Pachos (2012), Rao (2001)) will confirm that indeed appeals to a two dimensional topological structure is the standard explanation in the literature and constitutes part of our "best scientific theories."
} 
Moreover, we have to appeal to abstract mathematical structure, the fundamental group as the braid group $B_{N}$, in order to explain a physical phenomenon, specifically, fractional statistics (as they arise in physical FQHE systems). It is important to note that said structure is essentially abstract. It is solely in two dimensions that the fundamental group is the braid group. In three dimensions the structure allowing for fractional statistics disappears.

Hence, it seems that the explanation of anyons is in essence a mathematical and structural explanation, since it is the topological structure of the configuration space (of identical particles) that allows for fractional statistics. Moreover, such a case fits well with similar explanatory notions including “asymptotic explanation" (Batterman 2002), "topological explanation" (Nounou 2003), and "minimal model explanation" (Batterman and Collins 2014) in the following manner: these accounts call for an explanation of a general pattern portrayed by phenomena, instead of explanations of particular instances of pattern (as one may gain with a standard deductive-nomological or causal-mechanistic account of explanation). ${ }^{25}$ In the context of our discussion, the reason that all FQHE system can manifest fractional statistics, irrespective of the many varying details (apparatus, materials, varying parameters, initial and boundary conditions, etc.), is that in all such systems are represented with the same topological structure.

Connecting with Leng's $(2010,2012)$ notion of structural explanation we may say that (a) physical FQHE systems instantiated the structure corresponding to the configuration space of identical particles in two-dimensions (since there is little to no motion in the third spatial direction), and that (b) the existence of fractional statistics is a consequence of two facts: (i) The phase factor characterizing quantum statistics is the one-dimensional unitary representation of the fundamental group of a system's configuration space and (ii) in two dimensions the fundamental group is the Braid group. However, and importantly, it is no longer clear that we can dispense with reifying the explanatory mathematical structure. The reason is that, although FQHE systems are approximately two-dimensional, and although the do seem to manifest behavior consistent with fractional statistics; still, it is not the case that the mathematical structure is "approximately instantiated," as Leng's account necessitates. The fundamental group of the configuration space of identical particles in approximately two dimensions is the same as that of three dimensions, the permutation group $S_{N}$. In order to allow for fractional statistics we need the fundamental group to be the Braid group $B_{N}$, and this can only occur in

\footnotetext{
${ }^{25}$ See Hempel and Oppenheim (1948) for the deductive-nomological account of explanation and see, for example, Salmon (1984) and Woodward (2003) for causal accounts.
} 
exactly two dimensions. Yet, physical systems are not exactly two-dimensional. Thus, it cannot be said that a physical system "approximately instantiates" the Braid group structure necessary for fractional statistics. Said differently, by appealing to Leng's expounded account via Norton's distinction, the anyon case study is an example of a mismatch between a property of a limit system, i.e., a two-dimensional system with fundamental group that is the Braid group $B_{N}$, and the corresponding physical limit property, i.e., the fundamental group as the permutation group $S_{N}$. It is only in two dimensions that the fundamental group is the Braid group $B_{N}$, which makes room also for anyons with their fractional quantum statistics. Since there is a lack of correspondence between the property of the limit system and the limit property, our condition of adequacy from above is not satisfied. Hence, one can no longer talk about the mathematical structure being approximately instantiated by the physical system. It is then not clear how, on Leng's approach, one could dispense with reification in this context without undertaking the hard road to nominalism.

One may perhaps expect Leng to anticipate the type of objection raised here by an indispensable infinitesimal idealization. Reflecting on Maddy's (1997) discussion of the continuum model of fluids as an indispensable idealization, Leng (2010,120-121) says:

... we have a case of an essential idealization. . If this is right, then it looks as if the hypothesis that there are ideal, presumably abstract, fluids about which our theory of fluid dynamics asserts truths is indispensable to our best theoretical account of the behavior of physical fluids. . . But hold on a minute! Aren't our theoretical assumptions about ideal fluids still rather like our theoretical assumptions about ideal point masses or ideal frictionless planes? Shouldn't we expect our understanding of the success of our theory of ideal fluids to be analogous to our understanding of the success of these other theories? ... Given that we know of cases where the success of a theory is not due to the actual existence of the ideal objects it hypothesizes, but rather due to the way in which these convenient "myths" allow us to represent some facts about real objects, is this not enough to throw into question the claim that the existence of ideal continuous fluids should be considered as confirmed by the success of fluid dynamics?

It seems to me that, unless one can successfully apply a developed NSR account, such as the expounded version of Leng's approach, then the default answers to such questions-in the context of the case study of anyons and fractional statistics that I consider-ought to be "no." 


\section{Nominalistic Objections}

I have argued that the case of fractional statistics includes a genuine mathematical explanation that appeals indispensably to a mathematical structure that cannot be dispensed with via talk about "approximate instantiation." In this section I wish to consider three nominalist objections.

\subsection{First Objection: The Dilemma}

One may object that my account in this paper faces the following dilemma: Either physical systems approximately instantiate the structure needed to derive fractional statistics according to the standard story (given in Section 4) — whatever may be meant by "approximate instantiation" —or not. If so, then Leng's easy road nominalism succeeds. If not, then I have only succeeded in showing that the standard explanatory story regarding the emergence of anyons via the configuration space framework is deficient. In reply, let us consider each horn in turn. First, I have noted that the notion of "approximate instantiation" is vague, and have shown that when we expound on that account using Norton's wellreceived distinction between approximation and idealization, we learn that physical systems cannot approximately instantiate the braid group structure needed to derive anyons. It is now the burden of the defender of Leng-style NSR to offer a legitimate non-ad hoc characterization of "approximate instantiation." Without such a characterization, the default position ought to be that Leng's path to easy road has been blocked. Having said that, it is legitimate to consider why physicists think that systems portraying the FQHE are approximately two-dimensional in an effort to salvage Leng's account. Since it is conceded that actual FQHE systems portraying fractional statistics are actually three-dimensional, the claim then is that the systems are "dynamically two-dimensional" in the sense that motion in the thirddimension is quantized and constrained. In other words, it is possible to create systems in the laboratory that are dynamically, effectively two-dimensional, or even one- or zero-dimensional. The reason is that, in accordance with the third law of thermodynamics, all degrees of freedom freeze out in the limit of zero temperature. This means that we can approximately confine electrons to two-dimensional surfaces, one-dimensional lines, or even to zero-dimensional points, if we have a strongly confining potential and/or sufficiently low temperatures such that the excitation energy in one or more directions may be greater than the average thermal energy of the particles. Consider an illustration with a twodimensional electron gas such as in the context of the quantum Hall effects:

The electrons are confined to the surface of a semiconductor by a strong electric field, and they move more or less freely along the surface. On the other hand, the energy $E$ required to excite motion in the direction perpendicular to the surface is of the order of several milli-electron-Volt $(\mathrm{meV})$. Now at a temperature of say $T=1 K$, the thermal energy is $k T$, where $k$ is the Boltzmann constant. Thus if the transverse excitation energy is say $10 \mathrm{meV}$, then the fraction of electrons in the lowest excited transverse energy level is $e^{-\frac{E}{k T}}=e^{-100} \approx 10^{-44}$, which is zero for all practical purposes. (Khare 2005, 2) 
Prominent examples of dynamically two-dimensional systems include carriers confined to the vicinity of junctions between insulators and semiconductors, between layers of different semiconductors, and between vacuum and liquid helium, as well as layer compounds, intercalated graphite, and thin films.

Nevertheless, and in reply, these systems are not exactly two-dimensional so that truly twodimensional systems are idealizations or fictions of sorts. Ando et al. $(1982,439)$ explains:

These [dynamically, approximately two-dimensional] systems are not two-dimensional in a strict sense, both because the wave functions have a finite spatial extent in the third dimension and because electromagnetic fields are not confined to a place but spill out into the third dimension. Theoretical prediction for idealized two-dimensional systems must therefore be modified before they can be compared with experiment.

Perhaps, exact dynamically two-dimensional systems could be concocted at absolute zero temperature. The problem is that, in accordance with the third law, unless you have infinite time and resources, it is impossible to reach absolute zero temperature. This result was recently derived in Masenes and Oppenheim (2017). All this is to emphasize that systems that are dynamically two-dimensional, such as systems manifesting the quantum Hall effects, are not exactly two-dimensional-there is always some non-zero probability of an excitation in the third dimension.

But why do we need the system to be exactly, dynamically two-dimensional and not just twodimensional "for all practical purposes?" Recall our derivation of anyons from Section 4.1. On the configuration space approach to permutation invariance in $Q M$, anyons arise when the fundamental group of the configuration space is the braid group and this happens only for two-dimensions: $\pi_{1}(Q)=$ $B_{N}$ for $d=2$. For a three-dimensional system that is only effectively two-dimensional the fundamental group is the symmetry group, i.e., $\pi_{1}(Q)=S_{N}$ for $d=3$, and thus the mathematical structure is not rich enough to give rise to anyons and fractional statistics. Analogously, considering the toy-example from Section 3, it is only when we reach the limit system and have a two-dimensional square that we have a non-simply connected space and a non-trivial fundamental group. For any three-dimensional cuboid, no matter how close the height is to zero, we have a simply connected space and a trivial fundamental group.

To be clear, it is not assumed that the configuration space of a system is the physical space that it occupies. Whatever one thinks about the ontology of configuration space and its relation to fundamental physical space, at the very least the configuration space of a system represents the possible ways that particles can be arranged in physical space, i.e., the possible dynamics of the system. 
If a system's configuration space is two-dimensional, then the probability that one will find a particle located in the third dimension in physical space is zero. But the probability that one will find a particle in the third dimension in physical space in systems that manifest the FQHE is not zero (although it is extremely low, e.g., on the order of $\left.10^{-44}\right){ }^{26}$

Regarding the second horn, which rejects the standard explanatory story, what is important here is to note the motivation for such a rejection. Insofar as the motivation is to save Leng's approach, then the charge of explanatory deficiency is no more than question begging: if the standard explanatory story of the emergence of anyons does not support Leng-style nominalism, then so much the worse for Leng's approach. Similarly, if the motivation for rejecting the standard story is that it does not bode well with one's favorite account of scientific explanation, so much worse for one's favorite account. The viewpoint advocated in this paper is that "we should take the actual explanatory practices of scientists seriously when constructing our philosophical accounts of scientific explanation" (Bokulich 2008, 223-224). Likewise, the "job of philosophers of science and mathematics is to help make sense of, and contribute to, science and mathematics as practiced. The role of philosophers is not to overrule the pronouncements of mathematics and science on philosophical grounds ..." (Colyvan 2012b, 78; original emphasis).

Last, one's motivation may have to do with what we take to be a justification for an appeal to idealizations. The idea is that if there is a mismatch between a property of a limit system and a corresponding limit property, so that the condition of adequacy of Section 4 is not satisfied, then we are not justified in appealing to the property of the limit system in accounting or explaining some phenomenon of interests. Perhaps future work in mathematical physics will allow us to make sense of notions such as "approximately non-simply connected" or "approximate Braid group structure," or perchance we will discovery distinct ways of deriving anyonic behavior, at which point Leng's approach to easy road nominalism will succeed. Until then, we lack a justified explanation of anyons and

\footnotetext{
${ }^{26}$ An anonymous referee presses further: One wonders where the actual explanation arises. What seems to be doing the explaining is that the phase factors are what they are. There is of course a connection between the braid group and the phase factors - the approximately two-dimensional character of the configuration space removes the extra constraints on phase factors that arise in three-dimensions, constraints that prevent fractional statistics. Otherwise what is the motivation for thinking that the best explanation for what is going on here is the mathematical fact appealed to? My reply is that, first, it is the two-dimensional character of the configuration space-not the "approximately" two-dimensional character-that removes the constraint on phase factors arising in three-dimensions. Second, this is the only explanation of the emergence of anyons-the sole (developed) account of their theoretic possibility (to my best knowledge) - and the received explanation among the scientific community. I argue that if we take this explanation at face value it blocks Leng's approach to easy road nominalism. I do not deny that an easy road nominalist could reject the standard story and seek an alternative one, e.g., via future theory.
} 
fractional statistics. Generally, I am sympathetic to this line of reasoning (Shech (2015)). However, it must be noted that it is conjectural and depends on speculations regarding what shape future scientific theory may take. One may certainly retort as above and note that, first, appeals to an indispensable and essentially idealized topological structure constitute the main ingredient in the received explanatory story of fractional statistics. Second, as philosophers we need to take our current best science seriously and, thus, the default position ought to be that Leng's pathway to easy road nominalism has been blocked.

\subsection{Second Objection: An Anomaly}

One may worry that the case study that I have discussed is sufficiently narrow in scope and specially chosen so as not to warrant much attention. It is an anomaly and not part of the repertoire of our best scientific theories, so the claim goes. This is not the case.

First, although the phenomena of fractional statistics and the FQHE have been largely ignored by both philosophers of mathematics and science, the same is not true of the scientific community. For instance, publications having to do with the quantum Hall effect have averaged at about one publication per day as of 1995 (von Klitzing 2004). Moreover, insofar as the explanation of fractional quantum statistics is topological in nature, since it is an appeal to an idealized and abstract topological structure that is doing the explanatory work, it is only one instance of many such "topological effects." ${ }^{27}$ For example, the well-known phenomenon of the Aharonov-Bohm (AB) effect (Ahararonov and Bohm 1959) is standardly identified as topological in nature. ${ }^{28}$ The effect concerns a shift in an interference pattern, manifested by a beam of electrons in double-slit experiments, due to presence of an isolated magnetic field. In this context, the mathematical structure playing an indispensable explanatory role is the topological structure of the electron configuration space. However, in analogous manner to the case of fractional statistics, it is not clear that the mathematical structure can be approximately instantiated by the physical structure. This is so because it is standardly claimed that the

\footnotetext{
${ }^{27}$ As texts with titles such as Topological Effects in Quantum Mechanics (Afanasiec 1999) and Introduction to Topological Quantum Computation (Pachos 2012) confirm. In fact, the 2016 Nobel Prize in Physics was awarded to David J. Thouless, F. Duncan M. Haldane, and J. Michael Kosterlitz for theoretical discoveries of topological phase transitions and topological phases of matter.

${ }^{28} \mathrm{It}$ is of special interest to philosophers because it seems to portray a type of quantum non-locality (since the magnetic field affects a beam of electrons while being confined to a region where the beam is not) and raises a host of interpretive issues regarding ontology and indeterminism in classical electromagnetism and quantum mechanics. See, for example, Healey (2007), Maudling (1998), Mattingly (2006) (and references within) for some of the philosophical literature.
} 
$A B$ effect can only occur in a non-simply connected configuration space, but the configuration space corresponding to the $A B$ effect setup as it arises in the laboratory is not non-simply connected nor approximately non-simply connected (for the reasons stated in Section 3). ${ }^{29}$

One may object that the $A B$ effect is not topological. For instance, Katanaev (2011) puts forth a "geometric" interpretation of the AB effect. Moreover, Noble laureate Robert Laughlin's (1983) original account of the FQHE appeals to localization in the form of fractionally charged quasiparticles interacting with impurities in order to explain (part of) the FQHE and, importantly, it has been argued that fractional statistics are the results of an $A B$ effect-type phase picked up by the particles when they circle each other (Arovas et al. 1984). If the AB effect can be interpreted as a non-topological effect, then can't the FQHE, along with anyons and fractional statistics, be explained without appeals to topology? My reply comes in three parts. First, although I'm very much sympathetic to the idea that the $A B$ effect is not topological in nature it turns out to be non-trivial to argue for such a position (as is clear from Earman (2017) and my own Shech $(2015,2017))$ and, in any case, this is not the well-received standard interpretation. Second, the problem is exacerbated in the context of anyons and fractional statistics since, as emphasized above, the explanatory story of the emergence of anyons told in Section 4.1 shows that fractional statistics arise from the topological braid group structure. In fact, both Laughlin (1983) and Arovas et al. (1984) assume that they are working with a exactly two-dimensional system, and in such systems we know that anyons can emerge. Third, I am not arguing that nominalists cannot appeal to an alternative non-topological explanation of anyons, fractional statistics, and the FQHE. This option is certainly open to the nominalist and amounts to what I called the "second horn" (in Section 5.1). What I am saying is that such an explanation is conjectural and depends on speculations regarding what shape future scientific theory may take.

Further, setting aside effects identified as "topological," the problems for nominalists (of the NSR kind) raised in this paper may arise generally in instances where an appeal to an idealization that is indispensable for the purpose of account for some physical phenomenon. ${ }^{30}$ One example widely discussed includes the standard characterization of phase transition (such as a steaming cup of coffee) by our best scientific theories. ${ }^{31}$ In brief, phase transitions arising in some physical system are

\footnotetext{
${ }^{29}$ This claim is commonly found in both the scientific (e.g., Aharonov and Bohm $(1959,490)$, Wu and Yang (1975, 3854 ), Peshkin and Tonomura $(1989,27)$, Ryder $(1996,102))$ and philosophical (e.g. Batterman (2003), Nounou (2003)) literature. It is rejected by, e.g., Healey (2007), Earman (2017), Shech (2017).

${ }^{30}$ Batterman (2002) and Bokulich (2008) discus such examples.

${ }^{31}$ Such theories include mean-field theory, Yang-Lee's theory, Landau's approach and renormalization group methods. For more on the philosophical debate revolving around phase transitions see Batterman (2005),
} 
mathematically represented by a non-analytic partition function pertaining to the system. ${ }^{32}$ However, for any system with finite many particles (degrees of freedom) the partition function is analytic. The standard solution is to take an infinite limit in which the number of particles and volume of the system diverges, but the density remains constant. It is then claimed by authoritative figures that the "existence of a phase transition requires an infinite system. No phase transitions occur in systems with a finite number of degrees of freedom" (Kadanoff 2000,38). "This idealization is necessary because only infinite systems exhibit sharp phase transitions" (Ruelle 2004, 2). This type of example, which differs from standard appeals to idealizations, merits attention from philosophers of mathematics partaking in the easy road nominalism debate because it is no longer clear that one can wave away commitment to abstract mathematical structure with talk about "nominalistic content" and "approximate instantiation."

\subsection{Third Objection: Carrying the Explanatory Load}

Recently, Baron (2016) has attempted to break the explanatory symmetry between idealizations and mathematics by noting that, although idealizations play an indispensible explanatory role in our best sciences, they do not carry the explanatory load (while abstract mathematical structure may carry the explanatory load). His idea is that we can identify which indispensable explanatory parts of our scientific explanations carry the explanatory load via a difference making account cashed out in terms of counterfactuals (Baron 2016, 372):

Explanatory load A statement $\mathrm{S}$ that is (i) about some Fs and (ii) is a part of an explanation $\mathrm{E}$ of some phenomenon $\mathrm{P}$ is helping to carry the explanatory load iff $\mathrm{F}$ makes a difference to $\mathrm{P}$.

Difference-making $\mathrm{F}$ makes a difference to $\mathrm{P}$ iff had $\mathrm{F}$ not been the case, $\mathrm{P}$ would not have been the case.

To being, I think de Bianchi (2016) has adequately replied to Baron (2016). I take it that one of her main points is that Baron's (2016) account seems to presuppose that we can distinguish between idealized objects, properties, systems, models, etc., one the one hand, and abstract mathematical (but

Butterfield (2011), Menon and Callender (2013), Norton (2012), and Shech (2013). See Kadanof (2000) for a standard introductory text and Ruelle (2004) for rigorous results.

${ }^{32}$ Where by a non-analytic function we mean, roughly, that it is not possible to differentiate the function indefinitely, i.e., the function contains 'kinks' or 'discontinuities.' 
not idealized) objects, properties, systems, models, etc., on the other hand. However, our ability to make such distinction is not well supported by examples from mathematical physics and so Baron's account does not seem to extend to such examples. My hope is that the example of anyons and fractional quantum statistics helps to elucidate this point.

Nonetheless, if we assume that we can make the type of distinctions needed for Baron's account, say, between an idealized two-dimensional system and the abstract (but non-idealized) topological Braid group structure (associated with the fundamental group of the configuration space of identical particles in two-dimensions), it is still not clear to me that Baron's account succeeds in breaking the explanatory symmetry. Instead, it seems that on the standard explanatory story of anyons one needs both the abstract mathematical structure that is the Braid group and also the consideration of an idealized two-dimensional system. Within the two-dimensional setting, we can derive the Braid group structure which, in turn, allows us to derive fractional quantum statistics. Without the twodimensional setting, no such derivation is possible. There is then a sense for which both the idealization and abstract structure are "difference makers," even though actual systems that manifest fractional quantum statistics via the FQHE are not two-dimensional. Similar statements can be made regarding Baron's $(2016,374)$ comments on phase transitions:

[W] hen trying to explain why a phase transition occurs inside a boiling kettle, the number of $\mathrm{H}_{2} \mathrm{O}$ molecules makes no difference. Evidence for this comes from the many kettles that have been successfully boiled and phase transitions successfully induced. These kettles display a large variation in the number of $\mathrm{H} 2 \mathrm{O}$ molecules, whilst in each case a phase transition occurs anyway, so long as there is sufficient heat to produce boiling.

But on the standard scientific accounts of phase transitions, there can be no phase transitions in a system with any finite number degrees of freedom so that the number of $\mathrm{H}_{2} \mathrm{O}$ molecules is a difference maker for the phenomenon of phase transitions according to our best theories. This is why phenomena such as phase transitions, spontaneous symmetry breaking (in quantum statistical mechanics and quantum field theory), fractional quantum statistics, etc., are so puzzling and interesting: the standard characterization and explanation of such phenomena are at odds with what we take to be actualized in the world, namely, finite as opposed to infinite systems, three-dimensional as opposed to twodimensional systems, etc. To just note, as Baron does above, that the number of $\mathrm{H}_{2} \mathrm{O}$ molecules makes no difference to actual, concrete phase transitions in the world (which, in fact, is not the case since even 
phenomenologically we need a certain minimum amount of molecules for a phase transition), or that the non-two-dimensionality makes no difference to actual, concrete fractional quantum statistics manifested in the FQHE within the laboratory, misses the entire point!

Said differently, if by "difference maker" Baron (2016) means something that can make a causal difference to the occurrence of a concrete physical phenomenon, then no abstract object (mathematical or idealized) whatsoever can ever satisfy the role of difference maker. However, if by "difference maker" we mean making a difference to the mathematical derivation which is an essential ingredient in the explanatory story told by our best sciences of a some concrete physical phenomenon, then both the number of $\mathrm{H}_{2} \mathrm{O}$ molecules and the dimensionality of a system are difference makers for phase transitions and fractional statistics, respectively.

\section{Conclusion}

My goal in this essay was to make some interesting connections between the literature on infinite and infinitesimal idealizations in science and issues in philosophy of mathematics such as mathematical indispensability and explanation. In particular, I argued that the standard explanatory story of the phenomena of fractional statistics constitutes a genuine mathematical explanation that bears negatively on Leng's proposed path to easy road nominalism - specifically, in its expounded form via Norton's distinction between approximation and idealization. In doing so I take myself to have partially defended Mark Colyvan's claim that there is no easy road to nominalism. Having said that, I do not mean to be only critical of Leng's approach: I take it that fleshing out "approximate instantiating" via Norton's distinction is a positive development of any Leng-style NSR account. Future work in this direction may include looking to other examples of infinite/infinitesimal idealization in science and assessing whether or not they support Leng-style NSR.

More generally though, I aim to draw further attention to the possible explanatory symmetry between essential idealizations and mathematical objects. To that effect, consider again Baker's (e.g. 2005; 2009, 613) Enhanced Indispensability Argument (EIA), but with reference to mathematical objects extracted:

(1) We ought rationally to believe in the existence of any entity that plays an indispensable explanatory role in our best scientific theories.

(2) X plays an indispensable explanatory role in science. 
(3) Hence, we ought rationally to believe in the existence of $X$.

Concentrating on examples that arise in the literature on essential, infinite/infinitesimal idealization in physics (e.g., Batterman (2002, 2005), Bokulich (2008)), it seems that both "mathematical objects" and "idealizations" (or "idealized systems/models") could satisfy the role that "X" plays in the EIA. Those who take this point to be a kind of reductio against the EIA may exult if they hold nominalist intuitions, or try to break the symmetry (as Baron (2016) does) if they embrace Platonist intuitions. Alternatively, if one is willing to commit to the existence of abstract objects such as sets, numbers, fundamental groups, structure, etc., one may be willing also embrace ontological commitment to some idealized models such as infinite systems, two-dimensional systems, thermodynamic reversible processes, etc., which seem to be indispensible to, as well as part and parcel of, our best scientific theories. My suggestion then is that proponents of the easy road nominalism debate, and the debate on mathematical explanation, may benefit from attending to examples of essential, infinite/infinitesimal idealization science. Equally, it is would be interesting to explore whether accounts of scientific explanation such as Batterman (2002), Batterman and Rice (2014), and Bokulich (2008), which maintain that idealization and fictions are indispensible for explanatory purposes, ought to be committed to some form of Platonism because of the EIA. I leave such explorations for future work.

\section{Appendix A: The Configuration Space Approach to Fractional Quantum Statistics}

Let the configuration space of a particle in $d$-dimensions be $\mathbb{R}^{d}$ (where $\mathbb{R}$ is the set of real numbers) so that the position of the particles is given by an element of the space $x \in \mathbb{R}^{d}$. Consider $N$ such identical particles. The configuration space approach argues that the appropriate configuration space $Q$ for $N$ identical particles is not the Cartesian product of the single particle spaces, $\mathbb{R}^{N d} \equiv \mathbb{R}^{d} \times \ldots \times \mathbb{R}^{d}(N$ times), as one would expect if the particles were not identical. Instead, since the particles are identical, configurations that differ only by a permutation of particles ought to correspond to the same physical state. For the simplest case $d=1 N=2$, this means that the two configurations $\left(x_{1}, x_{2}\right)=(1,2)$ and $\left(x_{1}, x_{2}\right)=(2,1)$ actually represent the same state, and so we must divide out such permuted configurations. In other words, we move from the entire space $\mathbb{R}^{N d}=\mathbb{R}^{2 * 1}=\mathbb{R}^{2}$ of the twodimensional plane to consider only half the plane. In the context of the general case, this corresponds to 
considering the quotient (or identification) space that one attains by diving out the permutation group $S_{N}: Q=\frac{\mathbb{R}^{d}}{S_{N}}$.

Next, we'll want to excise the set $\Delta$ of diagonal points in $\mathbb{R}^{N d}$, which represent all points where the particles coincide. For the $d=1 N=2$ case, this means that we must excise points of the sort: $\left(x_{1}, x_{2}\right)=(x, x)$, where $x_{1}=x_{2}$. If we do not excise coincidence points (known as singular points) the resulting configuration space will not have a structure rich enough to represent fermions (or anyons).

We are left with a configuration space for $N$ identical particle in $d$ dimensions, in which we have divided out the action of the permutation group and excluded diagonal points: $\frac{\mathbb{R}^{d}-\Delta}{S_{N}}$. The exclusion of the diagonal point implies that the space in not simply connected and I denote this new configuration space with $\widetilde{Q}$. We must now undertake QM on a multiply connected space. In order to do so I will follow Morandi (1992, 114-144) closely.

Ordinary QM (in the Schrodinger picture) on simply connected regions represents the states of physical systems by complex wave functions $\Psi$ which are elements of a Hilbert space $\mathcal{H}$ of squareintegrable functions on the configuration space $Q$ over the field of complex numbers $\mathbb{C}: \Psi \in \mathcal{H}=$ $L_{\mathbb{C}}^{2}(Q)$, where $\mathcal{H}^{N}=L_{\mathbb{C}}^{2}(Q) \times \ldots \times L_{\mathbb{C}}^{2}(Q) \cong L_{\mathbb{C}}^{2}\left(Q^{N}\right)$ for $N$ identical particles. In order to extend ordinary QM to multiply connected regions we need to appeal the topological notion of a universal covering space $Q$ of $\widetilde{Q}$. That is to say, for any topological space, including ones that are not simply connected such as $\widetilde{Q}$, we can construct a universal covering space $Q$ that is simply connected (so that ordinary QM applies) with a covering projection map: $\pi: Q \rightarrow \widetilde{Q}$ (where $q \in Q$ and $\tilde{q} \in \widetilde{Q}$ ).

Since all the physical information in QM is contained in the squared modulus of the wave function (i.e. the probability density) $|\Psi(q)|^{2}$, we will want this quantity to be projectable down to $\widetilde{Q}$ for any $q \in Q$ in the sense that $|\Psi(q)|^{2}$ will depend only on a point $\tilde{q}=\pi(q)$ in the multiply connected configuration space $\widetilde{Q}$. If this condition is satisfied we say that we have a projectable quantum mechanics, and we will have succeeded in extending ordinary QM to a multiply connected region. To that effect, consider an arbitrary point $\tilde{q} \in \widetilde{Q}$, let $\tilde{C}$ be a closed curve beginning and ending at $\tilde{q}$, and let $[\tilde{C}]$ be the corresponding (first) homotopy class of curves based at $\tilde{q}$ so that $[\tilde{C}] \in \pi_{1}(\tilde{Q}, \tilde{q}) .{ }^{33}$ Further, let $q=\pi^{-1}(\tilde{q})$ be any point in the fiber over $\tilde{q}$. The homotopy lifting theorem says that all curves $\tilde{C}$ in $[\tilde{C}]$ are lifted to a curve $C$ in $Q$ beginning at $q$ and ending at some point $q^{\prime}$ that is also in the fiber over $\tilde{q}$ (so

\footnotetext{
${ }^{33}$ First, remember that $\pi_{1} \neq \pi: \pi_{1}$ represent the fundemantal group (the first homotopy group) and $\pi$ the covering projection. Second, roughly, recall that any two closed curves (loops) that can be continuously deformed into one another will be part of the same homotopy class. Homotopy is an equivalence relation among loops.
} 
that $\pi\left(q^{\prime}\right)=\tilde{q}$ and $\left.q^{\prime} \in Q\right) .{ }^{34}$ Denote this result by $q^{\prime}=[\tilde{C}] \cdot q$ and recall that $\gamma$ represents a phase factor (although, as of now, we have placed no constraints on the form of $\gamma$ ). We then have the following two central theorems (Morandi 1992, 119-120):

Theorem 1. Projectable Quantum Mechanics are obtained if and only if the wave functions on the universal covering space obey the boundary conditions:

$$
\begin{gathered}
\Psi([\tilde{C}] \cdot q)=\gamma([\tilde{C}]) \Psi(\mathrm{q}) \text { for all } q \in Q \\
\text { Where }|\gamma([\tilde{C}])|=1 \text { for all }[\tilde{C}] \in \pi_{1}(\tilde{Q})
\end{gathered}
$$

This means that wave functions on the universal covering space at different points (e.g. $q$ and $q^{\prime}$ ), but on the same fiber above some point in the multiply connected configuration space (e.g. $\tilde{q}$ ), can differ at most by a phase factor of modulus 1 . Moreover, since the universal covering space is simply connected, the wave functions must be single valued. We then get:

Theorem 2. The map $\gamma: \pi_{1}(\tilde{Q}) \rightarrow U(1)$ by $[\tilde{C}] \rightarrow \gamma([\tilde{C}])$ is a one-dimensional unitary representation of $\pi_{1}(\widetilde{Q})$.

Accordingly, we get our main result: In order to ascertain what type of phase factor is gained by a wave function when it is permuted-with the corresponding available quantum statistics-we must enquire into the one-dimensional unitary representation of the fundamental group of the configuration space of the system. Recall that in the case of $N$ identical particles in $d$ dimensions we have:

$$
\widetilde{Q}=\frac{\mathbb{R}^{d}-\Delta}{S_{N}}
$$

It has been shown by Artin (1947), Fadell and Neuwrith (1962), and Fox and Neuwrith (1962) that the fundamental group for the two- and three-dimensional cases are given by:

$$
\pi_{1}(\widetilde{Q})=B_{N} \text { for } d=2
$$

\footnotetext{
${ }^{34} \mathrm{C}$ is not necessarily a closed curve. If the bundle is not curved than $C$ is closed but if the bundle is curved than $C$ is not closed.
} 


$$
\pi_{1}(\widetilde{Q})=S_{N} \text { for } d=3
$$

Where $S_{N}$ is the permutation group and $B_{N}$ is the Braid group. Moreover, as stated previously, the onedimensional representation of $S_{N}$ is $\gamma= \pm 1,+1$ for bosons and -1 for fermions, while the onedimensional representation of $B_{N}$ is $\gamma_{(\theta)}=e^{i \theta}$ where $0 \leq \theta \leq 2 \pi$ so that the exchange phase can take on a continuous range of factors allowing for bosons, fermions and anyons.

Acknowledgements: I gratefully acknowledge useful discussions with Mary Leng and Mark Colyvan. Previous versions of this paper were presented at the "Annual Meeting of the European Philosophy of Science Association" at Exeter University on 09/09/2017 and "Current Projects" at Department of Philosophy at University of Sydney on 08/03/2017. I thank the participants for helpful comments. This work was produced as part of a visiting fellowship at the Sydney Centre for the Foundations of Science and Ideas and the Sydney Centre for Time at University of Sydney.

\section{References}

Afanasiev, G. N. 1999: Topological Effects in Quantum Mechanics. Kluwer Academic Publishers, Norwell, MA, USA.

Aharonov, Y., Bohm, D. 1959: Significance of electromagnetic potentials in the quantum theory. Phys. Rev. 115, 485-91.

Ando, T., Fowler, A. B., Stern, F. 1982: Electronic Properties of Two-dimensional Systems. Rev. of Mod. Phys. 54 437-672.

Arovas, D., P., Schrieffer, J. R., Wilczek, F. 1984: Fractional Statistics and the Quantum Hall Effect. Phys. Rev. Lett. 53 722-723.

Artin, E. 1947: Theory of Braids. Ann. Math. 48(1), 101-126.

Azzouni, Jody 2004: Deflating Existential Consequence: A Case for Nominalism.New York: Oxford University Press.

Azzouni, Jody 2012: 'Taking the Easy Road Out of Dodge'. Mind, 121, pp. 951-66.

Bain (2013) "Emergence in effective field theories." European Journal for Philosophy of Science, 3, 257273.

Bain (2016) "Emergence and the Mechanism in the Fractional Quantum Hall Effect" Studies in History and Philosophy of Modern Physics. 
Baker, Alan 2005: 'Are there Genuine Mathematical Explanations of Physical Phenomena?' Mind, 114, pp. 223-38.

Baker, Alan 2009: 'Mathematical Explanation in Science'. British Journal for the Philosophy of Science, 60, pp. 611-33.

Baker, Alan 2012: 'Science-Driven Mathematical Explanation'. Mind, 121, pp. 243-67.

Balaguer, Mark 1998: Platonism and Anti-Platonism in Mathematics. New York: Oxford University Press.

Batterman, Robert 2002: The Devil in the Details: Asymptotic Reasoning in Explanation, Reduction, and Emergence. Oxford University Press, London.

Batterman, Robert 2003: Falling cats, parallel parking, and polarized light. Stud. Hist. Philos. Mod. Phys. 34, 527-557.

Batterman, R. 2005: Critical phenomena and breaking drops: Infinite idealizations in physics. Stud. Hist. Philos. Mod. Phys. 36B, 225-44.

Batterman, R. and Collins, R. 2014: Minimal Model Explanations. Philosophy of Science, Vol. 81, No. 3 pp. 349-376.

Belot, G. 1998: Understanding electromagnetism. Br. J. for Philos. Sci. 49(4), 531-555.

Bokulich, A. 2008: Re-examining the Quantum-Classical Relation: Beyond Reductionism and Pluralism. Cambridge University Press.

Boyd, Richard N. 1983. On the current status of the issue of scientific realism. Erkenntnis 19:45-90.

Bueno, Otávio 2012: 'An Easy Road to Nominalism'. Mind, 121, pp. 967-82.

Butterfield, Jeremy 2011: 'Less is Different: Emergence and Reduction Reconciled'. Foundations of Physics. 41, 1065-1135.

Camino, F. E., Zhou, W., Goldman, V. J. 2005: Realization of a Laughlin Quasiparticle Interferometer: Observation of fractional statistics. Phys. Rev. B 72075342.

Chakraborty, T. and Pietilinen, P. [1995]: The Quantum Hall Effects, Berlin: Springer.

Colyvan, Mark 2001: The Indispensability of Mathematics. New York: Oxford University Press.

Colyvan, Mark 2007: 'Mathematical Recreation Versus Mathematical Knowledge', In Leng, Paseau, and Potter 2007, pp. 109-22.

Colyvan, Mark 2010: 'There is No Easy Road to Nominalism'. Mind, 119, pp. 285-306.

Colyvan, Mark 2012a: 'Road Work Ahead: Heavy Machinery on the Easy Road'. Mind, 121, pp. 10311046. 
Colyvan, Mark 2012b. An Introduction to the Philosophy of Mathematics. Cambridge: Cambridge University Press.

Duck, I., Sudarshan, E. C. G. (eds.) 1997: Pauli and the Sping-statistics Theorem. World Scientific, Singapore.

Earman, John 2010: “Understanding permutation invariance in quantum mechanics." Unpublished Preprint.

Earman, John 2017: "The Role of Idealizations in the Aharonov-Bohm Effect." Synthese https://doi.org/10.1007/s11229-017-1522-9

Ehrenberg, W., Siday, R. W. 1949: The refractive index in electron optics and the principles of dynamics. Proc. Phys. Soc. London B62, 8-21.

Ezawa Z. F. 2013: Quantum Hall Effects. World Scientific, Singapore.

Fadell, E., Neuwirth, L. 1962: Configuration Spaces. Math. Scand. 10, 111-118.

Field, Hartry H. 1980: Science Without Numbers: A Defense of Nominalism. Oxford: Blackwell.

Fox, R., Neuwirth, L. 1962: The Braid Groups. Math Scand. 10, 119-126.

Fradkin, E.(2013). Field theories of condensed matter physics. (2nd ed.) Cambridge: CUP.

Frigg, R. 2006. "Scientific Representation and the Semantic View of Theories," Theoria 55, 2006, 49-65.

Guay, A. and Sartenaer, O. [2016a]: "A new look at emergence. Or when after is different." European Journal for Philosophy of Science, 6: 297-322.

Guay, A. and Sartenaer, O. [2016b]: "Emergent Quasiparticles. Or how to get a rich physics from a sober metaphysics." Forthcoming in: O. Bueno, R. Chen \& M. B. Fagan (eds.) Individuation across Experimental and Theoretical Sciences, Oxford University Press: Oxford, 2017. Doi: http://hdl.handle.net/2078.1/179059

Halperin, B. I. 1984: Statistics of Quasiparticles and the Hierarchy of Fractional Quantized Hall States. Phys. Rev. Lett. 52 1583-1586.

Hatcher, A.: Algebraic Topology. Cambridge University Press (2002)

Healey, R. A. 2007: Gaugin What's Real: The Conceptual Foundations of Contemporary Gauge Theories. Oxford University Press, New York. 
Hempel, C., and Paul. Oppenheim 1948: "Studies in the Logic of Explanation." Philosophy of Science 15:135-75. Repr. in Aspects of Scientific Explanation and Other Essays in the Philosophy of Science, ed. C. Hempel (New York: Free Press, 1965), 245-90.

Hughes, R. I. G. (1989). "Bell's theorem, ideology, and structural explanation" In Cushing and McMullin (Eds.) Philosophical Consequences of Quantum Theory: Reections on Bell's Theorem. University of Notre Dame Press. 195-207.

Khare, A. 2005: Fractional Statistics and Quantum Theory. World Scientific, NJ, USA.

Klitzing, K. v. 2004: 25 Years of Quantum Hall Effect (QHE): A Personal View on the Discovery, Physica and Application of this Quantum Effect. In: Douçot, B., Pasquier, V., Duplantier, B., Rivasseau, V. (eds.) The Quantum Hall Effect Poincaré Seminar, vol. 45, 1-23 Birkhäuser Verlag, Basel Boston Berlin.

Laidlaw, M. G., DeWitt, C. M. 1971: Feyman Functional Integrals for System of Indistinguishable Particles. Phys. Rev. D 3 1375-1378.

Lancaster, T., \& Pexton, M. (2015). "Reduction and emergence in the fractional quantum Hall state." Studies in History and Philosophy of Modern Physics, 52, 343-357.

Landsman, N. P. 2016. "Quantization and superselection sectors III: Multiply connected spaces and indistinguishable particles." Rev. Math. Phys., 28, 1650019

Laughlin, R. (1983) 'Anomalous Quantum Hall Effect: An Incompressible Quantum Fluid with Fractionally Charged Excitations', Physical Review Letters 50, 1395-8.

Lederer, P. (2015). "The quantum Hall effects: Philosophical approach." Studies in History and Philosophy of Modern Physics, 50, 25-42.

Leinaas, J. M., Myrheim, J. 1977: On the Theory of Identical Particles. Nuovo Cimento B 37 1-23.

Leng, Mary. 2005. "Mathematical Explanation" in Cellucci, Carlo and Donald Gillies (eds), Mathematical Reasoning and Heuristics. London: King's College Publications.

Leng, Mary 2010: Mathematics and Reality. Oxford: Oxford University Press.

Leng, Mary 2012: 'Taking it Easy: A Response to Colyvan'. Mind, 121, pp. 983-96.

Leng, Mary, Alexander Paseau, and Michael Potter (eds) 2007: Mathematical Knowledge. Oxford: Oxford University Press.

Liggins, David 2013: 'Weaseling and the Content of Science'. Mind, 121, pp. 997-1006.

Maddy, P. 1997. Naturalism in Mathematics. Oxford: Clarendon Press. 
Masenes, L., Oppenheim, J. 2017: "A general derivation and quantification of the third law of thermodynamics." Nature Communications 8, 14538.

Mattingly, J. 2006: Which Gauge Matters. Stud. Hist. Philos. Mod. Phys. 37, 243-262.

Maudlin, T. 1998: Discussion: Healey on the Aharonov-Bohm effect. Philos. of Sci., 65, 361-368.

Melia, Joseph 2000: 'Weaseling Away the Indispensability Argument'. Mind, 109, pp. 455-7.

Melia, Joseph 2002: 'Reply to Colyvan'. Mind, 111, pp. 75-9.

Menon, T., and C. Callender. 2013. "Turn and Face the Strange ... Ch-Ch-Changes: Philosophical Questions Raised by PhaseTransitions." In The Oxford Handbook of Philosophy of Physics, ed. R. W. Batterman. Oxford: Oxford University Press.

Messiah, A. M., Greenberg, O. W. 1964: Symmetrization Postulate and its Experimental Foundation. Phys. Rev. B 136 248-267.

Morandi, G. 1992: The Role of Topology in Classical and Quantum Mechanics. Springer-Verlag, Berlin.

Niiniluoto, Ilkka. 1999. Critical Scientific Realism. Oxford: Oxford University Press.

Norton, John D. 2012: 'Approximations and Idealizations: Why the Difference Matters.' Philosophy of Science 79, 207-32.

Nounou, A, 2003: A Fourth Way to the Aharonov-Bohm Effect. In: Bradind, K., Castellani, E. (eds.) Symmetries in Physics: Philosophical Replections, Cambridge University Press.

Pachos, J. K. 2012: Introduction to Topological Quantum Computation. Cambridge University Press, Cambridge, UK.

Pauli, W. 1940: 'The connection between spin and statistics.' Phys. Rev. 58, 716-722.

Peshkin, M., Tonomura, A.1989: The Aharonov-Bohm Effect. LNP, vol. 340. Springer, Berlin.

Psillos, Stathis. 1999. Scientific Realism: How Science Tracks Truth. London: Routledge.

Putnam, Hilary 1971: Philosophy of Logic. New York: Harper.

Quine, Willard, V. O. 1981b: Theories and Things. Cambridge, MA: Harvard University Press.

Railton, P. 1980. Explaining Explanation: A Realist Account of Scientific Explanation and Understanding. Ph.D. Dissertation, Princeton University.

Rao, S. 2001. “An Anyon Primer.” Available via arXiv:hep-th/9209066

Ruelle, David. 2004: Thermodynamic Formalism. 2nd ed. Cambridge: Cambridge University Press. 
Ruetsche, Laura 2011: Interpreting Quantum Theories: The Art of the Possible. Oxford University Press.

Ryder, L. H. 1996: Quantum Field Theory. Cambridge University Press, Cambridge.

Salmon, Wesley. 1984: Scientific Explanation and the Causal Structure of the World. Princeton, NJ: Princeton University Press.

Shech, E. 2013. "What is the 'Paradox of Phase Transitions?'" Philosophy of Science, 80 (December 2013): 1170-1181.

Shech, E. 2015. "Two Approaches to Fractional Statistics in the Quantum Hall Effect: Idealizations and the Curious Case of the Anyon" Foundations of Physics, Volume 45, Issue 9: 1063-110.

Shech, E. 2017. "Idealizations, Essential Self-Adjointness, and Minimal Model Explanation in the Aharonov-Bohm Effect." Synthese, 1-25.

Stern, A. 2008. "Anyons and the quantum hall effect-a pedagogical review." Ann. Phys. 323, 204-249.

Tonomura, A. 1999: Electron Holography. Springer-Verlag, Berlin Heidelberg New York.

Tonomura, A. 2010: The AB Effect and its Expanding Applications. J. Phys. A: Math. Theor. 43 1-13.

Wilczek, F. 1982a: Magnetic Flux, Angular Momentum and Statistics. Phys. Rev. Lett. 48 1144-1146.

Wilczek, F. 1982b: Quantum Mechanics of Fractional-Spin Particles. Phys. Rev. Lett. 49 957-959.

Wilczek, F. (ed.) 1990: Fractional Statistics and Anyon Superconductivity. World Scientific, Singapore.

Woodward, James F. 2003. Making Things Happen: A Theory of Causation. Oxford: Oxford University Press.

Wu, Y. S. 1984: General Theory for Quantum Statistics in Two Dimensions. Phys. Rev. Lett. 52 2103-2106.

Wu, T. T. and C. N. Yang 1975: Concept of Nonintegrable Phase Factors and Global Formulation of Gauge Fields. Phys. Rev. D12, 3845.

Yablo, S. 1998: 'Does Ontology Rest on a Mistake?' Aristotelian Society, Supplementary Volume, 72, pp. 229-61.

Yablo, S. 2002: 'Abstract Objects: A Case Study'. Philosophical Issues, 12, pp. 220-40.

Yablo, S. 2012: 'Explanation, Extrapolation, and Existence'. Mind, 121, pp. 1007-30. 